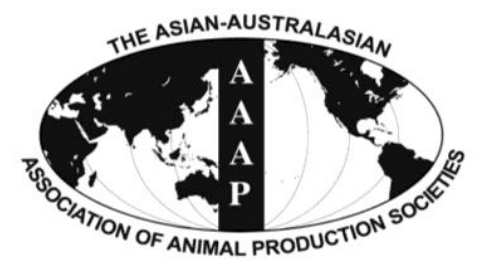

Asian-Aust. J. Anim. Sci.

Vol. 26, No. 3 : 366-377 March 2013

http://dx.doi.org/10.5713/ajas.2012.12551

www.ajas.info

pISSN 1011-2367 elSSN 1976-5517

\title{
Nutritive Value of Grasses in Semi-arid Rangelands of Ethiopia: Local Experience Based Herbage Preference Evaluation versus Laboratory Analysis
}

\author{
Habtamu T. Keba ${ }^{1}$ *, I. C. Madakadze ${ }^{1}$, A. Angassa ${ }^{2}$ and A. Hassen ${ }^{3}$ \\ ${ }^{1}$ Faculty of Natural and Agricultural Sciences, Department of Plant Production and Soil Science, \\ University of Pretoria, P. Bag X20 Hatfield, Pretoria, 0028, South Africa
}

\begin{abstract}
We examined the nutritive value of common grass species in the semi-arid rangelands of Borana in southern Ethiopia using local experience based herbage preference (LEBHP) perception and laboratory techniques. Local pastoralists in the study area were asked to identify common grass species and rank them according to the species' preferences and palatability to cattle. The pastoralists listed a total of 15 common grass species which were then sampled during the main rain and cold dry seasons and analyzed for crude protein (CP), Neutral Detergent Fiber (NDF), Acid Detergent Fiber (ADF) and ash content to verify pastoralists' claim regarding the quality of individual species. The relative feed value (RFV) and dry matter digestibility (DMD) were also calculated using NDF and ADF contents. Spearman's rank correlation was used to examine possible relationships between laboratory results and pastoralists' experience on grass quality. Cenchrus ciliaris, Chrysopogon aucheri, Digitaria milanjiana, Eragrostis papposa and Panicum maximum were the top five species based on LEBHP perception. There were indications of inconsistency in terms of LEBHP perception among the different pastoral communities. The chemical composition of all grass species showed significant $(\mathrm{p}<0.05$ ) variation between sites, seasons and species. The results showed that the $\mathrm{CP}$ values for the Borana rangelands were in the range of $8.7 \%$ in the main rain season to $5.1 \%$ for the cold dry season. The fiber constituents were relatively low in the main rain season compared to the cold dry season. Overall, Digitaria milanjiana had the highest CP (16.5\%) content, while the least was recorded with Heteropogon contortus (10.8) and Aristida adoensis (9.8\%) during the main rain season. It seems that the spatial variability of landscapes within the wider geographical regions, soil properties and texture, and land-use patterns probably contributed to site differences in species quality. Generally, the RFV of individual grass species was significantly $(p<0.05)$ varied between and within sites. The ranking of species by pastoralists according to their preferences by cattle was highly correlated with the chemical composition of laboratory results of individual grass species with ' $r$ ' values for CP (0.94), ash (0.95), NDF (-0.98), ADF (-0.93) and ADL (-0.93). We suggest the complimentary use of LEBHP and laboratory techniques in evaluating the nutritive quality of rangeland forage species for sustainable animal production. (Key Words: Forage Preference Ranking, Grass Nutritive Value, Relative Feed Value, Semi-arid Rangelands, Spatial Variation in Feed Quality)
\end{abstract}

\section{INTRODUCTION}

Herbaceous species play an important role in livestock feeding in arid and semi-arid regions (Arzani et al., 2006) and also improve ecosystem services for the welfare of pastoral societies (Stoddart et al., 1975). In general,

\footnotetext{
* Corresponding Author: Habtamu T. Keba. Tel: +27-12-420-

3667, Fax: +27-12-420-4120, E-mail: h.teka@yahoo.com

2 School of Animal and Range Sciences, Hawassa University, private Bag, 1591, Awassa, Ethiopia.

${ }^{3}$ Deparment of Animal Science and Wilde Life, University of Pretoria, Agriculture Building 10-32, South Africa.

Submitted Oct. 5, 2012; Accepted Dec. 10, 2012; Revised Dec. 17, 2012
}

production of herbaceous biomass is primarily determined by the amount, distribution and duration of rainfall. Recently, most pastoral areas of Ethiopia, including the Borana rangelands, are exhibiting a shift from herbaceous species to woody plants, a feature that is accompanied with some degree of degradation resulting from overgrazing, expansion of cultivation and settlement (Oba, 1998; Gemedo- Dalle et al., 2006; Angassa and Oba, 2010). This is up and above the general decline in forage biomass yield due to changes in the amount and distribution of rainfall. Both internal and external pressures on the rangeland-use policy have influenced the environmental soil characteristics and changes in the vegetation composition 
and diversity (Oba et al., 2008). The expansion of cultivated areas has been increased nutrient leaching from the soil and run off from the rangelands. This has the potential of reducing the nutrient content and nutritive value of herbaceous plants, an aspect that has implications on livestock production and livelihood of pastoralists.

The Borana rangelands in southern Ethiopia are used for communal grazing and extensive livestock production system with natural grazing as the main feed base. The Borana pastoralists have been practicing transhumance to counter seasonal fluctuations in forage and water resources. A recent study (Angassa and Oba, 2010) has highlighted shifts in species composition and declines in dry matter production of herbaceous plants. These ecological changes would also imply changes in the nutritive value of the rangeland plants. The factors that have been reported to affect the nutrient value of herbaceous plants are seasonal variability (Snyman, 1998), species variation (Arzani et al., 2008), soil nutrient status of production location (Tessema et al., 2011), grazing pressure (Henkin et al., 2011) and management aspects (van der Westhuizen et al., 2005). In the semi-arid Borana, this translates into exacerbated seasonal shortages and low nutritive value of the available forage (Alemayehu, 2006) further hindering growth and sustainable livestock production.

A recent literature (Ganskopp and Bohnert, 2001) has shown that knowledge of the nutritional dynamics of rangeland forage species is important to sustain satisfactory growth and reproduction of livestock without deterioration of rangeland. Such awareness further assists in planning for proper utilization and to envisage nutrient deficiencies, a basis for suggesting supplemental requirements for animals (Arzani et al., 2006). While formal ecological assessments and interpretations are fine there is usually a gap between the traditional and formal interpretations of changes occurring in the field. The Borana pastoralists have been known to exist since before the thirteenth century (Oba and Kotile, 2001) and have adapted to the local situation and manage their land in such a way as to be able to survive and develop there without destroying it. Their traditional strategies for rangeland management have been reported to be superior to the approaches used by modern ecologists. The LEBHP evaluation that allows for this evolves through adaptive processes and is handed down through generations by cultural transmission (Beever et al., 2000). In terms of access to forage, pastoralists would have significant insights into the shortage of forage resources as a result of rainfall variability, rangeland shrinkage and degradation, and the changes in the quality of common forage species.

This study was conducted as part of a larger project on the ecological changes in the Borana rangelands in southern Ethiopia. For this component, pastoralists' experiences and perceptions of forage species and changes in quality were mirrored against laboratory based assessments. The objectives of the study were to: i) determine the nutritive value of the common indigenous grass species in Borana using both LEBHP perceptions and laboratory analysis, and ii) evaluate seasonal and spatial variability of the nutritive value of the common grass species.

\section{MATERIAL AND METHODS}

\section{Study sites}

The study was conducted in Oromia Regional State of Ethiopia, Borana Zone between March and August 2010. The region is dominated by arid and semi-arid climate that is characterized by high temperature and bimodal type of rainfall. The mean annual rainfall is about $500 \mathrm{~mm}$ (Angassa and Oba, 2007), while the mean annual temperature varies from $19^{\circ} \mathrm{C}$ to $24^{\circ} \mathrm{C}$ with little seasonal variation based on elevation (Coppock, 1994). The average plant growing days vary from 100 to 140 in the west and north of the study areas, respectively. This corresponds to 1.5 to 2.0 ton DM/ha/year of herbaceous forage production. The Borana rangelands have perennial herbaceous cover that is interrupted in place by woody vegetation that is dominated by Acacia and Commiphora species (Coppock, 1994). The soils of the rangelands were derived from sedimentary and volcanic materials and the soil texture ranges from sandy, silt to clay (Gemedo-Dalle et al., 2006).

\section{Sampling procedure}

Five communal grazing areas and two ranches were selected following discussions with district experts and knowledgeable community representatives. The communal grazing areas selected for sample collection included: Surupha, Did-Hara, Mana-Soda, Medhacho and Bokkulboma. The two ranches were Did-Tuyura, a government ranch located $17 \mathrm{~km}$ northeast of Yabello town and operated under the Oromia Pastoral Area Development Commission; and Dambala-Wachu ranch which is located about $55 \mathrm{~km}$ south of Yabello town and is a cooperative ranch managed by the community. The selected study areas represented different histories of land use and grazing intensities. Two communal grazing areas (Surupha, DidHara) and one of the ranches (Did-Tuyura) are located in the former wet season grazing areas and upper semi-arid zone (Coppock, 1994). Mana-Soda, Medhacho and Bokkulboma and Dambala-Wachu ranch were located in the former dry season grazing areas, which are also classified as the lower semi-arid zone. Wet season grazing areas refers to those portions of rangelands utilized only during the rainy season due to lack of surface water, while dry season grazing areas are rangelands associated with permanent deep-well water points. Generally, the concepts of wet and dry season grazing areas have been abandoned due to the 
expansion of sedentarization and crop cultivation in the rangelands. Land-use management and the slope of these sites were also taken into consideration to select comparable grazing areas for the purpose of this study. After establishing a $10 \mathrm{~km}$ transects at each site, 10 main plots $(20 \mathrm{~m} \times 50 \mathrm{~m})$ were marked at $1 \mathrm{~km}$ intervals. These were used for sampling the herbaceous species.

The common grass species were selected and sampled based on the relative abundances and pastoralists' experiences of preferences on each grass species. The samples were collected in two seasons viz: during the main rain season (March to May, 2010) and cold dry season (July to August, 2010). At the time of vegetation sampling, five quadrats of $0.5 \mathrm{~m} \times 0.5 \mathrm{~m}$ were randomly placed within the previously marked main plots and used for grass species collection. Common grass species found within the $0.5 \mathrm{~m} \times$ $0.5 \mathrm{~m}$ sampling quadrat were identified with the participation of pastoralists and hand clipped using a sickle at ground level, weighed and oven dried at $105^{\circ} \mathrm{C}$ for $48 \mathrm{~h}$. The dried samples were ground to pass through a $1 \mathrm{~mm}$ screen at Debere-Zeit, Ethiopian Agricultural Research Center Laboratory. All samples were kept in airtight containers before chemical analysis. Soil samples were also collected from each main plot along the established transects using an auger up to a depth of $30 \mathrm{~cm}(0$ to $30 \mathrm{~cm})$. The soil samples were air dried and kept in plastic bags for laboratory analysis.

\section{Perception analysis}

Data for analysis of perceptions of pastoralists was collected at the same time during sample collection on herbaceous plants. Seven community level group discussions were held at a village level in each study site (Mana-Soda, Did-Tuyura, Medhacho, Bokulboma, DidHara, Surupha, and Dambala-Wachu) to select key local informants. Selection was based on the individual's indigenous knowledge and ability to feedback the issues and experiences during the exercise to the parent group. Pastoral communities from each study site selected 10 respondents, giving a total of 70 respondents across the seven locations, for the identification and ranking of species based on their preferences by livestock. Then, the selected respondents participated in the ranking of the same common grass species used for laboratory analysis. Species preference ranking was according to LEBHP and importance for a particular livestock type. Individual interviews were followed by group discussions with respondents' groups at each study location. A semistructured questionnaire was administered to elderly community leaders and three agricultural extension agents at each study site. The cumulative match of respondents' perception of prioritizing a particular species across the study sites was divided by the total number of respondents (i.e. 70) and multiplied by 100 to obtain the percentage value for that particular species. Then, all the species were ranked in descending order based on their percentage value in relation to other species under investigation. The perception value of individual species was then correlated with the results of the chemical composition of that particular species using Spearman's rank correlation (Fowler and Cohen, 1996).

\section{Grass chemical analysis}

The ash and nitrogen contents of individual grass species were analyzed using the standard procedures of AOAC (1990). Nitrogen content was determined by the micro-Kjeldahl method, while crude protein (CP) was calculated by multiplying the nitrogen content with a factor of 6.25. Acid detergent fiber (ADF), neutral detergent fiber (NDF) and acid detergent lignin (ADL) in the forage samples were determined using the method of Van Soest et al. (1990). The relative feed value (RFV) was calculated according to Stallings (2005) using the following procedure:

$$
\begin{aligned}
\mathrm{RFV}= & (\text { Dry matter digestibility }(\mathrm{DMD}) \\
& \times \text { Dry matter intake }(\mathrm{DMI})) / 1.29
\end{aligned}
$$

Where $1.29=$ the expected digestible dry matter intake as $\%$ of body weight; $\mathrm{DMD}=83.58-0.824 \times \mathrm{ADF} \%+2.626$ N\% after Oddy et al. (1983); DMI $=120 / \%$ NDF.

\section{Soil sample analysis}

Soil texture was analyzed using the hydrometer method (Gee and Bauder, 1986), while soil $\mathrm{pH}$ was read in a 1:2.5 soil-water suspension using a pH meter (McLean, 1982). Organic carbon was analyzed by oxidation with potassium dichromate $\left(\mathrm{K}_{2} \mathrm{Cr}_{2} \mathrm{O}_{7}\right)$ in a sulfuric acid medium, whereas, organic matter content was calculated by multiplying the value of organic carbon reading with a constant $(K=1.724)$. Total nitrogen was estimated by the Kjeldahl method (AOAC, 1991) and available phosphorus by the Bray method for soil samples with $\mathrm{pH}>7$ and the Olsen procedure for samples with $\mathrm{pH}<7$ (Olsen and Sommers, 1982). Cation exchange capacity (CEC) was measured after ammonium acetate (1 $\mathrm{N} \quad \mathrm{NH}_{4} \mathrm{OAC}$ ) extraction (Van Reeuwijk, 1995).

\section{Statistical analysis}

Descriptive statistics was used in summarizing and describing the survey data. We used season, species type and site differences as categorical predictor variables. Grass species nutritive values and soil parameters were considered as numerical response variables. The experimental design was completely randomized and analyzed as a three factor experiment (season, site and species). Data were subjected 
to analysis of variance using SAS version 9.2 (SAS institute, 2001). Correlation of dependent and independent variables were performed using SPSS version 17. Statistical significance was reported at $\mathrm{p}<0.05$.

\section{RESULTS}

\section{Variation in nutritive value of the grass species}

The relative frequencies of the collected grass species across all sites are presented in Figure 1. Chrysopogon aucheri, Cenchrus ciliaris and Eragrotis papposa had high frequencies of $>50 \%$. Six other species were of intermediate frequency $(20-25 \%$ ), while the rest six grass species had frequencies below 20\%. Overall, season, site and species type and their two and three-way interactions were significant $(P<0.01)$ in terms of ash, $\mathrm{CP}, \mathrm{NDF}$ and ADF contents. The results on chemical constituents are presented by site for each season in Table 1 to 4 .

There was some variation in crude protein content of the herbaceous species at Did-Tuyura, Dambala-Wachu and Surupha but this did not vary within site for the remaining sites (Table 1). Those species which showed relatively low CP content at Did-Tuyura site included: E. papposa, $C$. aucheri and Themeda triandra. Similarly, the CP recorded for Heteropogon contortus at Dambala-Wachu site was significantly $(\mathrm{p}<0.05)$ lower as compared to the CP of other grass species. Across sites and at species level only $C$. ciliaris, D. milanjiana, E. papposa and $H$. contortus showed a significant $(\mathrm{p}<0.05)$ variation in terms of CP (Table 1$)$. Overall, the highest $\mathrm{CP}$ content was recorded in

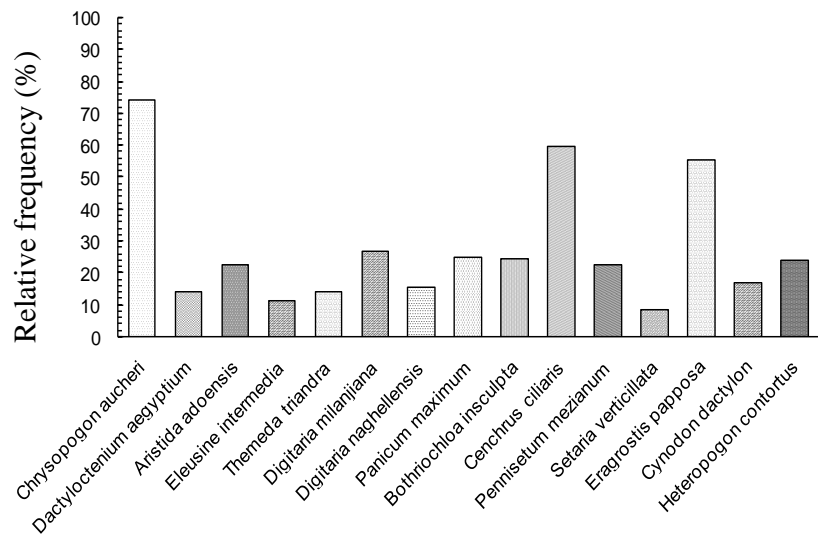

Figure 1. Relative frequency of herbaceous plants in the Borana rangelands of Ethiopia in the main rain season.

Bokkulboma as compared to the other sites. This was followed by values recorded for CP contents of samples from Did-Hara, Did-Tuyura, Mana-Soda, Surupha and Medhacho. We recorded a significantly $(\mathrm{p}<0.05)$ lowest average CP content at Dambala-Wachu. Within site, the ash content of grass species in the main rain season was significantly $(\mathrm{p}<0.05)$ varied among the grass species at Mana-Soda, Bokkulboma, Did-Hara, Surupha, DambalaWachu and Did-Tuyura. The grass species collected from Medhacho did not show variation in terms of ash content (Table 2). Across sites, the majority of the grass species exhibited significant $(\mathrm{p}<0.05)$ differences in their ash content. Themeda triandra, P. maximum and E. intermedia did not show any variation in terms of their ash content across the different sites. On average, the ash content

Table 1. Species variation in crude protein $(\%)$ across different sites in the Borana rangelands during the main rain season

\begin{tabular}{|c|c|c|c|c|c|c|c|c|}
\hline \multirow[b]{2}{*}{ Species } & \multicolumn{7}{|c|}{ Sites } & \multirow[b]{2}{*}{ LSD } \\
\hline & Did-Tuyura & Mana-Soda & Medhacho & Bokkulboma & Did-Hara & Surupha & $\begin{array}{c}\text { Dambala- } \\
\text { Wachu }\end{array}$ & \\
\hline Cenchrus ciliaris & $7.6^{\mathrm{abAB}}$ & $6.3^{\mathrm{aB}}$ & $5.7^{\mathrm{aB}}$ & $8.2^{\mathrm{aAB}}$ & $7.3^{\mathrm{aAB}}$ & $9.8^{\mathrm{aA}}$ & $6.1^{\mathrm{abB}}$ & 3.2 \\
\hline Chrysopogon aucheri & $5.4^{\mathrm{bA}}$ & $7.7^{\mathrm{aA}}$ & $6.4^{\mathrm{aA}}$ & $7.6^{\mathrm{aA}}$ & $6.6^{\mathrm{aA}}$ & $6.4^{\mathrm{abA}}$ & $6.9^{\mathrm{abA}}$ & 4.9 \\
\hline Digitaria milanjiana & $7.8^{\mathrm{abAB}}$ & $8.9^{\mathrm{aA}}$ & & $8.1^{\mathrm{aAB}}$ & $5.6^{\mathrm{aB}}$ & - & $7.4^{\mathrm{aAB}}$ & 2.9 \\
\hline Eragrostis papposa & $5.5^{\mathrm{bB}}$ & $5.6^{\mathrm{aB}}$ & $7.2^{\mathrm{aAB}}$ & - & $6.2^{\mathrm{aAB}}$ & $8.7^{\mathrm{abA}}$ & $5.3^{\mathrm{abB}}$ & 2.9 \\
\hline Panicum maximum & $7.7^{\mathrm{abA}}$ & $6.9^{\mathrm{aA}}$ & - & - & $6.3^{\mathrm{aA}}$ & $6.3^{\mathrm{abA}}$ & $6.2^{\mathrm{abA}}$ & 3.2 \\
\hline Themeda triandra & $5.4^{\mathrm{bA}}$ & - & - & - & $7.3^{\mathrm{aA}}$ & $7.1^{\mathrm{abA}}$ & - & 5.5 \\
\hline Heteropogon contortus & $6.2^{\mathrm{abAB}}$ & $6.2^{\mathrm{aAB}}$ & & $7.6^{\mathrm{aA}}$ & $6.1^{\mathrm{aAB}}$ & $5.5^{\mathrm{bBC}}$ & $3.8^{\mathrm{bC}}$ & 1.9 \\
\hline Bothriochloa insculpta & $5.5^{\mathrm{bA}}$ & $5.9^{\mathrm{aA}}$ & $8.7^{\mathrm{aA}}$ & $6.1^{\mathrm{aA}}$ & $5.8^{\mathrm{aA}}$ & $6.0^{\mathrm{abA}}$ & $6.0^{\mathrm{abA}}$ & 4.2 \\
\hline Eleusine intermedia & - & - & - & - & - & $6.8^{\mathrm{abA}}$ & $6.8^{\mathrm{abA}}$ & 1.7 \\
\hline Digitaria naghellensis & - & - & - & $6.8^{\mathrm{aA}}$ & $6.9^{\mathrm{aA}}$ & & $7.3^{\mathrm{aA}}$ & 4 \\
\hline Cynodon dactylon & $10.2^{\mathrm{aA}}$ & & $7.0^{\mathrm{aA}}$ & $11.18^{\mathrm{aA}}$ & $8.9^{\mathrm{aA}}$ & $9.4^{\mathrm{abA}}$ & - & 4.9 \\
\hline Dactyloctenium aegyptium & - & - & $6.6^{\mathrm{aA}}$ & $8.6^{\mathrm{aA}}$ & - & - & $4.7^{\mathrm{abA}}$ & 4.7 \\
\hline Setaria verticillata & - & - & $7.4^{\mathrm{aA}}$ & $7.4^{\mathrm{aA}}$ & - & - & - & 3.1 \\
\hline Pennisetum mezianum & - & $6.5^{\mathrm{aA}}$ & $7.4^{\mathrm{aA}}$ & $5.8^{\mathrm{aA}}$ & $6.1^{\mathrm{aA}}$ & - & $7.1^{\mathrm{abA}}$ & 1.9 \\
\hline Aristida adoensis & $6.5^{\mathrm{abA}}$ & - & - & - & $5.4^{\mathrm{aA}}$ & $5.9^{\mathrm{abA}}$ & - & 3.8 \\
\hline Mean & $6.8^{\mathrm{B}}$ & $6.9^{\mathrm{B}}$ & $7.1^{\mathrm{B}}$ & $8.0^{\mathrm{A}}$ & $6.6^{\mathrm{BC}}$ & $7.2^{\mathrm{B}}$ & $6.1^{\mathrm{C}}$ & 0.92 \\
\hline LSD & 4.5 & 4.4 & 3.6 & 6.5 & 5.4 & 4.2 & 3.3 & \\
\hline
\end{tabular}

Means in a column followed by different lower case letters (ab--etc) and upper case letters in a row (AB--etc) are significantly different ( $<<0.05)$. 
Table 2. Variation of ash content $(\%)$ among herbaceous species and across different sites in the Borana in the main rain season

\begin{tabular}{|c|c|c|c|c|c|c|c|c|}
\hline \multirow[b]{2}{*}{ Species } & \multicolumn{7}{|c|}{ Sites } & \multirow[b]{2}{*}{ LSD } \\
\hline & Did-Tuyura & Mana-Soda & Medhacho & Bokkulboma & Did-Hara & Surupha & $\begin{array}{c}\text { Dambala- } \\
\text { Wachu }\end{array}$ & \\
\hline Chrysopogon aucheri & $14.5^{\mathrm{aA}}$ & $13.9^{\mathrm{bcAB}}$ & $14.9^{\mathrm{aAB}}$ & $17.1^{\mathrm{bcA}}$ & $15.6^{\mathrm{abA}}$ & $11.1^{\mathrm{bB}}$ & $17.0^{\mathrm{aA}}$ & 4.2 \\
\hline Digitaria milanjiana & $14.3^{\mathrm{aAB}}$ & $13^{\mathrm{cB}}$ & - & $16.4^{\mathrm{bcdeA}}$ & $16.5^{\mathrm{abA}}$ & - & $13.1^{\mathrm{abcB}}$ & 3.1 \\
\hline Cynodon dactylon & $13.7^{\mathrm{abAB}}$ & - & $13.0^{\mathrm{aAB}}$ & $16.2^{\text {bcdeA }}$ & $13.4^{\mathrm{abAB}}$ & $11.5^{\mathrm{bB}}$ & - & 4.4 \\
\hline Themeda triandra & $13.5^{\mathrm{abA}}$ & - & - & - & $13.4^{\mathrm{abA}}$ & $12.6^{\mathrm{abA}}$ & - & 5.1 \\
\hline Heteropogon contortus & $13.2^{\mathrm{abAB}}$ & $15.4^{\mathrm{abcA}}$ & & $11.4^{\mathrm{eAB}}$ & $13.7^{\mathrm{abAB}}$ & $10.8^{\mathrm{bB}}$ & $13.3^{\mathrm{abcAB}}$ & 4.4 \\
\hline Cenchrus ciliaris & $12.4^{\mathrm{abA}}$ & $16.7^{\mathrm{aAB}}$ & $11.3^{\mathrm{aB}}$ & $19.2^{\mathrm{aA}}$ & $13.7^{\mathrm{abAB}}$ & $15.5^{\mathrm{abAB}}$ & $14.5^{\mathrm{abAB}}$ & 6.4 \\
\hline Eragrostis papposa & $11.6^{\mathrm{abB}}$ & $13.4^{\mathrm{bcB}}$ & $12.9^{\mathrm{aB}}$ & - & $18.9^{\mathrm{abA}}$ & $15.2^{\mathrm{abAB}}$ & $15.1^{\mathrm{abAB}}$ & 4.6 \\
\hline Panicum maximum & $11.2^{\mathrm{abA}}$ & $13.8^{\mathrm{bcA}}$ & - & - & $20.7^{\mathrm{aA}}$ & $20.6^{\mathrm{aA}}$ & $13.8^{\mathrm{abA}}$ & 13.1 \\
\hline Bothriochloa inscuipta & $11.1^{\mathrm{abB}}$ & $13.4^{\mathrm{bcB}}$ & $12.7^{\mathrm{aB}}$ & $11.9^{\mathrm{deB}}$ & $18.1^{\mathrm{abA}}$ & $11.1^{\mathrm{bB}}$ & $11.1^{\mathrm{bcB}}$ & 2.9 \\
\hline Aristida adoensis & $9.5^{\mathrm{bB}}$ & - & - & - & $21.9^{\mathrm{aA}}$ & $9.1^{\mathrm{bB}}$ & - & 3.9 \\
\hline Pennisetum mezianum & - & $13.7^{\mathrm{bcB}}$ & $13.6^{\mathrm{aB}}$ & $17.1^{\mathrm{bcA}}$ & $11.4^{\mathrm{cB}}$ & - & $14.7^{\mathrm{abA}}$ & 3.2 \\
\hline Dactyloctenium aegyptium & - & - & $14.6^{\mathrm{aAB}}$ & $16.7^{\mathrm{bcdA}}$ & $13.3^{\mathrm{abB}}$ & - & $12.1^{\mathrm{bcB}}$ & 3.4 \\
\hline Setaria verticillata & - & - & $15.1^{\mathrm{aA}}$ & $11.7^{\mathrm{deB}}$ & - & & - & 2.6 \\
\hline Digitaria naghellensis & - & - & - & $16.7^{\text {bcdeA }}$ & - & $10.6^{\mathrm{bA}}$ & $9.5^{\mathrm{cC}}$ & 1.9 \\
\hline Eleusine intermedia & - & - & - & - & - & $10.6 \mathrm{bA}$ & $11.6^{\mathrm{bcA}}$ & 2.3 \\
\hline Mean & $12.5^{\mathrm{D}}$ & $14.6^{\mathrm{BC}}$ & $13.9^{\mathrm{CD}}$ & $15.9^{\mathrm{AB}}$ & $16.7^{\mathrm{A}}$ & $12.8^{\mathrm{D}}$ & $13.6^{\mathrm{CD}}$ & 1.9 \\
\hline LSD & 4.7 & 3.3 & 3.9 & 5.1 & 8.7 & 9.1 & 4.5 & \\
\hline
\end{tabular}

Mean in columns with different lower case letters (ab--etc) and upper case in rows (AB--etc) are significantly different $(\mathrm{p}<0.05)$.

recorded at Did-Hara was the highest (16.7\%), while the lowest ash contents were recorded at Surupha $(12.8 \%)$ and Did-Tuyura (12.5\%).

Generally, the NDF content during the main rain season for the sampled herbaceous species was high $>70 \%$ (Table $3)$. There was a significant variation between sites in terms of NDF with the highest value at Did-Tuyura (76.8\%), while the lowest values were recorded at Medhacho (71.7) and Bokkulboma (71\%). Within site, the NDF content was generally significant among the different grass species with the exception of Mana-Soda site. The ranking of individual grass species within sites was also greatly differed. There were a few species that had NDF values below $70 \%$. These included: S. verticillata (65\%) at Medhacho; $S$. verticillata (67.2\%) and D. aegyptium (67.2\%) at Bokkulboma and $E$. papposa $(63.1 \%)$ at Surupha. Overall, across the study sites, the NDF content of T. triandra, A. adoensis, P.mezianum, $D$. aegyptium, S. verticillata and E. intermedia did not show

Table 3. Variation of neutral detergent fiber (\%) between species and different sites in the Borana in the main rain season

\begin{tabular}{|c|c|c|c|c|c|c|c|c|}
\hline \multirow[b]{2}{*}{ Species } & \multicolumn{7}{|c|}{ Sites } & \multirow[b]{2}{*}{ LSD } \\
\hline & Did-Tuyura & Mana-Soda & Medhacho & Bokkulboma & Did-Hara & Surupha & $\begin{array}{c}\text { Dambala- } \\
\text { Wachu }\end{array}$ & \\
\hline Chrysopogon aucheri & $78.9^{\mathrm{abAB}}$ & $77^{\mathrm{abAB}}$ & $76.8^{\mathrm{aAB}}$ & $73.2^{\mathrm{abB}}$ & $79.2^{\mathrm{abA}}$ & $79.8^{\mathrm{aA}}$ & $77.9^{\mathrm{abAB}}$ & 5.9 \\
\hline Digitaria milanjiana & $75.9^{\mathrm{abcA}}$ & $75.9^{\mathrm{abA}}$ & $77.3^{\mathrm{aA}}$ & $74.2^{\mathrm{abAB}}$ & $72.8^{\mathrm{cdB}}$ & - & $72.7^{\mathrm{bB}}$ & 2.9 \\
\hline Cynodon dactylon & $73.3^{\mathrm{bcA}}$ & - & - & $75.5^{\mathrm{abA}}$ & $77.5^{\mathrm{abcdA}}$ & $75.2^{\mathrm{abA}}$ & - & 8.5 \\
\hline Themeda triandra & $72.2^{\mathrm{cA}}$ & - & - & - & $75.3^{\mathrm{abcdA}}$ & $73.5^{\mathrm{abA}}$ & - & 4.2 \\
\hline Heteropogon contortus & $76.8^{\mathrm{abcAB}}$ & $78.9^{\mathrm{aA}}$ & & $71.6^{\mathrm{abB}}$ & $74.7^{\mathrm{abcdAB}}$ & $73.4^{\mathrm{bB}}$ & $75.9^{\mathrm{abAB}}$ & 5.2 \\
\hline Cenchrus ciliaris & $77.8^{\mathrm{abcA}}$ & $74.6^{\mathrm{abB}}$ & $74.3^{\mathrm{aB}}$ & $75.2^{\mathrm{abAB}}$ & $74.9^{\mathrm{abcdAB}}$ & $76.6^{\mathrm{abAB}}$ & $74.5^{\mathrm{bB}}$ & 2.9 \\
\hline Eragrostis papposa & $77.3^{\mathrm{abcA}}$ & $76.7^{\mathrm{abA}}$ & $72.9^{\mathrm{aA}}$ & - & $73.9^{\mathrm{bcdA}}$ & $63.1^{\mathrm{cB}}$ & $74.1^{\mathrm{bA}}$ & 6.8 \\
\hline Panicum maximum & $77.3^{\mathrm{abcA}}$ & $78.0^{\mathrm{abA}}$ & - & - & $74.9^{\mathrm{abcdA}}$ & $74.9^{\mathrm{abA}}$ & $78.0^{\mathrm{abA}}$ & 11.2 \\
\hline Bothriochloa inscuipta & $76.7^{\mathrm{abc} A \mathrm{~B}}$ & $78^{\mathrm{abA}}$ & $77.9^{\mathrm{aA}}$ & $77.4^{\mathrm{aAB}}$ & $71.9^{\mathrm{dB}}$ & $77.1^{\mathrm{abAB}}$ & $75.4^{\mathrm{bAB}}$ & 5.8 \\
\hline Aristida adoensis & $81.4^{\mathrm{aA}}$ & - & - & - & $78.5^{\mathrm{abcA}}$ & $78.2^{\mathrm{abA}}$ & - & 4.3 \\
\hline Pennisetum mezianum & - & $73.6^{\mathrm{abA}}$ & $72.9^{\mathrm{aA}}$ & $72.1^{\mathrm{abA}}$ & - & - & $72.1^{\mathrm{bA}}$ & 5.2 \\
\hline Dactyloctenium aegyptium & - & - & $74.5^{\mathrm{aA}}$ & $67.2^{\mathrm{bA}}$ & - & - & $72.8^{\mathrm{bA}}$ & 7.9 \\
\hline Setaria verticillata & - & - & $65.0^{\mathrm{bA}}$ & $67.2^{\mathrm{bA}}$ & - & - & - & 8.4 \\
\hline Digitaria naghellensis & - & - & - & $78.9^{\mathrm{aB}}$ & $80.4^{\mathrm{aB}}$ & & $82.9^{\mathrm{aA}}$ & 2.1 \\
\hline Eleusine intermedia & - & - & - & - & - & $77.9^{\mathrm{abA}}$ & $75.7^{\mathrm{abA}}$ & 3.2 \\
\hline Mean & $76.8^{\mathrm{A}}$ & $75.4^{\mathrm{AB}}$ & $71.7^{\mathrm{C}}$ & $71.0^{\mathrm{C}}$ & $74.2^{\mathrm{B}}$ & $75.0^{\mathrm{AB}}$ & $74.8^{\mathrm{AB}}$ & 3.4 \\
\hline LSD & 6.2 & 12.1 & 6.7 & 8.4 & 6.1 & 6.4 & 7.1 & \\
\hline
\end{tabular}

Mean in columns with different lower case letters (ab--etc) and upper case in rows (AB--etc) are significantly different $(\mathrm{p}<0.05)$. 
Table 4. Variation of acid detergent fiber (\%) between species and different sites in the Borana in the main rain season

\begin{tabular}{|c|c|c|c|c|c|c|c|c|}
\hline \multirow[b]{2}{*}{ Species } & \multicolumn{7}{|c|}{ Sites } & \multirow[b]{2}{*}{ LSD } \\
\hline & Did-Tuyura & Mana-Soda & Medhacho & Bokkulboma & Did-Hara & Surupha & $\begin{array}{c}\text { Dambala- } \\
\text { Wachu }\end{array}$ & \\
\hline Cenchrus ciliaris & $49.2^{\mathrm{abcA}}$ & $47.0^{\mathrm{aAB}}$ & $43.6^{\mathrm{aB}}$ & $48.5^{\mathrm{abcAB}}$ & $45.9^{\mathrm{abAB}}$ & $43.5^{\mathrm{bcB}}$ & $45.1^{\mathrm{bcdAB}}$ & 4.5 \\
\hline Chrysopogon aucheri & $50.5^{\mathrm{abA}}$ & $46.7^{\mathrm{abA}}$ & $46.6^{\mathrm{aA}}$ & $47.6^{\mathrm{aA}}$ & $50^{\mathrm{abA}}$ & $47.8^{\mathrm{abA}}$ & $48.8^{\mathrm{abA}}$ & 4 \\
\hline Digitaria milanjiana & $46^{\mathrm{cdA}}$ & $41.8^{\mathrm{cC}}$ & & $45.0^{\mathrm{abcAB}}$ & $44.3^{\mathrm{bABC}}$ & - & $42.9^{\mathrm{cdBC}}$ & 3 \\
\hline Eragrostis papposa & $48.3^{\mathrm{bcAB}}$ & $45.0^{\mathrm{abcBC}}$ & $46.4^{\mathrm{aAB}}$ & - & $49.7^{\mathrm{abA}}$ & $41.1^{\mathrm{cC}}$ & $49.8^{\mathrm{abA}}$ & 4.4 \\
\hline Panicum maximum & $50.5^{\mathrm{abA}}$ & $43.5^{\mathrm{bcB}}$ & - & - & $51^{\mathrm{abA}}$ & $51 . .9^{\mathrm{aA}}$ & $43.5^{\mathrm{cdB}}$ & 6.3 \\
\hline Themeda triandra & $44.1^{\mathrm{deA}}$ & - & - & - & $48.2^{\mathrm{abA}}$ & $47.9^{\mathrm{abA}}$ & - & 4.1 \\
\hline Heteropogon contortus & $50.8^{\mathrm{abA}}$ & $47.7^{\mathrm{aAB}}$ & - & $45.9^{\mathrm{abc} A B}$ & $48.5^{\mathrm{abAB}}$ & $44.1^{\mathrm{bcB}}$ & $46.9^{\mathrm{abcAB}}$ & 5.9 \\
\hline Bothriochloa inscuipta & $53.4^{\mathrm{aA}}$ & $46.4^{\mathrm{abBC}}$ & $44.3^{\mathrm{aC}}$ & $46.5^{\mathrm{abBC}}$ & $48.5^{\mathrm{abB}}$ & $46.2^{\mathrm{abcBC}}$ & $46.2^{\mathrm{abcdBC}}$ & 3.4 \\
\hline Eleusine intermedia & - & - & - & - & - & $47.7^{\mathrm{abA}}$ & $45.1^{\mathrm{bcdB}}$ & 1.9 \\
\hline Digitaria naghellensis & - & - & - & $46.5^{\mathrm{abB}}$ & $47.7^{\mathrm{abB}}$ & - & $51.1^{\mathrm{aA}}$ & 2.7 \\
\hline Cynodon dactylon & $40.0^{\mathrm{eB}}$ & - & $46.2^{\mathrm{aA}}$ & $43.4^{\mathrm{bcAB}}$ & $46.6^{\mathrm{abA}}$ & $42.2^{\mathrm{abB}}$ & - & 3.5 \\
\hline Dactyloctenium aegyptium & - & $44.5^{\mathrm{aA}}$ & $44.5^{\mathrm{aA}}$ & $43.2^{\mathrm{bcA}}$ & - & - & $41.5^{\mathrm{dA}}$ & 5.6 \\
\hline Setaria verticillata & - & - & $44.9^{\mathrm{aA}}$ & $42.1^{\mathrm{cA}}$ & $48.9^{\mathrm{abA}}$ & - & $47^{\mathrm{abcA}}$ & 5.8 \\
\hline Pennisetum mezianum & - & $46.5^{\mathrm{abA}}$ & $47.2^{\mathrm{aA}}$ & $47.9^{\mathrm{aA}}$ & $47.9^{\mathrm{aA}}$ & $48.9^{\mathrm{abA}}$ & - & 4.2 \\
\hline Aristida adoensis & $51.7^{\mathrm{aA}}$ & - & - & - & $47.3^{\mathrm{abB}}$ & $47.7^{\mathrm{abB}}$ & - & 1.5 \\
\hline Mean & $48.5^{\mathrm{A}}$ & $45.7^{\mathrm{B}}$ & $45.3^{\mathrm{B}}$ & $45.5^{\mathrm{B}}$ & $48.1^{\mathrm{A}}$ & $46.0^{\mathrm{B}}$ & $46.2^{\mathrm{B}}$ & 1.8 \\
\hline LSD & 4.2 & 3.3 & 5.6 & 4.2 & 6 & 5.9 & 4.9 & \\
\hline
\end{tabular}

Mean in columns with different lower case letters (ab--etc) and upper case in rows (AB--etc) are significantly different $(\mathrm{p}<0.05)$.

Table 5. Variation of relative feed value for herbaceous species across the different sites

\begin{tabular}{|c|c|c|c|c|c|c|c|c|}
\hline \multirow[b]{2}{*}{ Species } & \multicolumn{7}{|c|}{ Sites } & \multirow[b]{2}{*}{ LSD } \\
\hline & Did-Tuyura & Mana-Soda & Medhacho & Bokkulboma & Did-Hara & Surupha & $\begin{array}{c}\text { Dambala- } \\
\text { Wachu }\end{array}$ & \\
\hline Cenchrus ciliaris & $48.1^{\mathrm{cdB}}$ & $52.8^{\mathrm{aAB}}$ & $56.9^{\mathrm{aAB}}$ & $52.7^{\mathrm{dAB}}$ & $53.1^{\mathrm{bAB}}$ & $53.1^{\mathrm{abAB}}$ & $54.9^{\mathrm{abcA}}$ & 6.0 \\
\hline Chrysopogon aucheri & $47.0^{\mathrm{cdA}}$ & $50.8^{\mathrm{aA}}$ & $51.7^{\mathrm{cA}}$ & $52.6^{\mathrm{dA}}$ & $47.1^{\mathrm{cA}}$ & $48.7^{\mathrm{bA}}$ & $48.4^{\mathrm{cdA}}$ & 7.5 \\
\hline Digitaria milanjiana & $52.2^{\mathrm{bcB}}$ & $55.9^{\mathrm{aAB}}$ & - & $54.2^{\mathrm{cdAB}}$ & $57.2^{\mathrm{aA}}$ & - & $57.9^{\mathrm{abA}}$ & 3.9 \\
\hline Eragrostis papposa & $50.0^{\mathrm{cdB}}$ & $53.8^{\mathrm{aB}}$ & $54.3^{\mathrm{abB}}$ & - & $50.6^{\mathrm{abB}}$ & $69.4^{\mathrm{aA}}$ & $50.8^{\mathrm{bcdB}}$ & 10.4 \\
\hline Panicum maximum & $46.8^{\mathrm{cdA}}$ & $54.8^{\mathrm{aA}}$ & - & - & $47.4^{\mathrm{abA}}$ & $47.5^{\mathrm{bA}}$ & $54.8^{\mathrm{abcA}}$ & 10.3 \\
\hline Themeda triandra & $58.2^{\mathrm{abA}}$ & - & - & - & $50.5^{\mathrm{abB}}$ & $52.1^{\mathrm{bB}}$ & - & 5.9 \\
\hline Heteropogon contortus & $47.5^{\mathrm{cdB}}$ & $49.7^{\mathrm{aAB}}$ & & $55.5^{\mathrm{bcAB}}$ & $51.1^{\mathrm{abAB}}$ & $57.2^{\mathrm{bA}}$ & $53.3^{\mathrm{abc} A B}$ & 8.1 \\
\hline Bothriochloa insculpta & $45.5^{\mathrm{dB}}$ & $51.3^{\mathrm{aAB}}$ & $52.2^{\mathrm{cA}}$ & $51.6^{\mathrm{dA}}$ & $53.5^{\mathrm{abA}}$ & $52.2^{\mathrm{bA}}$ & $53.2^{\mathrm{abcA}}$ & 5.9 \\
\hline Eleusine intermedia & - & - & - & - & - & $49.6^{\mathrm{bB}}$ & $53.8^{\mathrm{abcA}}$ & 3.5 \\
\hline Digitaria naghellensis & - & - & - & $50.2^{\mathrm{dA}}$ & $48.0^{\mathrm{abA}}$ & - & $43.0^{\mathrm{dB}}$ & 4.7 \\
\hline Cynodon dactylon & $59.1^{\mathrm{aA}}$ & - & $51.9^{\mathrm{cAB}}$ & $53.4^{\mathrm{dAB}}$ & $50.3^{\mathrm{abB}}$ & $55.8^{\mathrm{bAB}}$ & - & 7.3 \\
\hline Dactyloctenium aegyptium & - & - & $55.7^{\mathrm{abA}}$ & $62.2^{\mathrm{abA}}$ & - & - & $60.7^{\mathrm{aA}}$ & 9.4 \\
\hline Setaria verticillata & - & - & $62.8^{\mathrm{aA}}$ & $64.3^{\mathrm{aA}}$ & - & - & - & 12.4 \\
\hline Pennisetum mezianum & - & $53.9^{\mathrm{aA}}$ & $53.4^{\mathrm{abA}}$ & $53.9^{\mathrm{cdA}}$ & $50.7^{\mathrm{abA}}$ & & $54.5^{\mathrm{abcA}}$ & 7.5 \\
\hline Aristida adoensis & $43.8^{\mathrm{dB}}$ & - & - & - & $50.4 \mathrm{abA}$ & $49.8^{\mathrm{bA}}$ & - & 1.7 \\
\hline$L S D$ & 6.3 & 14.2 & 10.1 & 8.4 & 10.1 & 10.1 & 8.7 & \\
\hline
\end{tabular}

Mean in columns with different lower case letters (ab--etc) and upper case in rows (AB--etc) are significantly different $(\mathrm{p}<0.05)$.

any significant variations, while the remaining grass species exhibited significant variations across sites.

The average ADF content across the study sites was $46.5 \%$ and there was significant $(\mathrm{p}<0.05)$ site and within site variation in ADF content of samples (Table 4). Nevertheless, herbaceous species such as C. aucheri, $T$. triandra, D. aegyptium, $S$. verticillata and $P$. mezianum did not demonstrate variation across sites. At Medhacho, the ADF content showed no significant variation among species type. There were significant $(\mathrm{p}<0.05)$ differences in terms of RFV among the grass species except at Mana-Soda (Table 5). Only C. aucheri, P. maximum, D. aegyptium and $S$. verticillata did not show significant $(\mathrm{p}>0.05)$ differences in terms of the RFV of common grasses across the study sites.

\section{Ranking of species by pastoralists compared to chemical analysis}

The ranking of the common grass species by Borana pastoralists is presented in Table 6. Cenchrus ciliaris, $C$. aucheri, D. milanjiana, E. papposa and $P$. maximum were ranked as the top five grass species in the region. Digitaria naghellensis, C. dactylon, D. aegyptium, S. verticillata, $P$. mezianum and $A$. adoensis were perceived as species of low preference and palatability. The remaining grass species were considered as moderately valuable/palatable species 
Table 6. Rank of herbaceous species according to Borana pastoralists' interest

\begin{tabular}{|c|c|c|c|c|c|c|c|c|c|}
\hline Herbaceous species & $\begin{array}{l}\text { Rank by } \\
\text { respondent }\end{array}$ & Did-Tuyura & $\begin{array}{l}\text { Dambala- } \\
\text { Wachu }\end{array}$ & Mana-Soda & Medhacho & Did-Hara & Surupha & $\begin{array}{c}\text { Bokkul } \\
\text { boma }\end{array}$ & $\begin{array}{l}\text { Frequency of } \\
\text { respondent } \%\end{array}$ \\
\hline Cenchrus ciliaris & 1 & 9 & 9 & 10 & 10 & 9 & 8 & 9 & 91 \\
\hline Chrysopogon aucheri & 2 & 9 & 8 & 8 & 9 & 9 & 7 & 8 & 83 \\
\hline Digitaria milanjiana & 3 & 8 & 8 & 7 & 8 & 7 & 6 & 7 & 73 \\
\hline Eragrostis papposa & 4 & 8 & 7 & 8 & 6 & 5 & 4 & 5 & 61 \\
\hline Panicum maximum & 5 & 7 & 6 & 5 & 6 & 5 & 6 & 5 & 57 \\
\hline Themeda triandra & 6 & 7 & 5 & 5 & 5 & 6 & 6 & 5 & 56 \\
\hline Heteropogon contortus & 7 & 6 & 6 & 5 & 5 & 6 & 5 & 5 & 54 \\
\hline Bothriochloa insculpta & 8 & 6 & 7 & 5 & 5 & 4 & 4 & 6 & 53 \\
\hline Eleusine intermedia & 9 & 6 & 5 & 5 & 4 & 6 & 5 & 5 & 51 \\
\hline Digitaria naghellensis & 10 & 5 & 4 & 6 & 5 & 4 & 4 & 6 & 49 \\
\hline Cynodon dactylon & 11 & 4 & 5 & 4 & 5 & 4 & 5 & 3 & 43 \\
\hline Dactyloctenium aegyptium & 12 & 5 & 4 & 4 & 3 & 4 & 5 & 4 & 41 \\
\hline Setaria verticillata & 13 & 5 & 4 & 5 & 4 & 3 & 4 & 4 & 41 \\
\hline Pennisetum mezianum & 14 & 4 & 4 & 5 & 6 & 3 & 3 & 3 & 40 \\
\hline Aristida adoensis & 15 & 3 & 0 & 0 & 0 & 3 & 5 & 4 & 21 \\
\hline
\end{tabular}

Notes: Rank by respondents 1-5 = best, $6-10=$ moderate, $11-15=$ worst.

(Table 6).

The correlation coefficients between the perception ranking and the chemical constituents of the common grass species are presented in Table 7. The ranking of individual grass species for $\mathrm{CP}$ and ash content based on our respondents' perceptions was positively correlated with the laboratory-based analysis of the same species both in the main rain and cold dry seasons. The structural constituents of NDF and ADF were negatively correlated with perception-based respondents' ranking in both seasons. Generally, the laboratory-based analysis confirmed pastoralists' experience and knowledge of forage quality and species preferences by a particular class of livestock. It seems that integration of these two sources of information can complement each other in future development endeavors.

\section{Seasonal effect on the nutritive value of herbaceous species}

Annual rainfalls variability on the study sites are presented in Figure 2. Based on the nearest weather station at Yabello, Did-Tuyura, Did-Hara and Surupha had a better rainfall than the other study sites. Accordingly, Moyale is the nearest weather station for Bokkulboma, which had a better rainfall than Mega station (Dambala-Wachu, Medhacho and Mana-Soda). The mean monthly rainfall ranged from 8 to $150 \mathrm{~mm}$ at Yabello area, 6.7 to $128.2 \mathrm{~mm}$ at Mega area, 5.3 to $144.6 \mathrm{~mm}$ for Bokkulboma. The

Table 7. Correlation coefficient between experience based herbage value rank and chemical composition determined by laboratory analyses

\begin{tabular}{|c|c|c|c|c|c|c|}
\hline \multirow{2}{*}{ Season } & \multirow{2}{*}{ Site } & \multicolumn{5}{|c|}{$\mathrm{r}$ value for } \\
\hline & & $\mathrm{CP}$ & Ash & $\mathrm{NDF}$ & $\mathrm{ADF}$ & ADL \\
\hline \multirow[t]{7}{*}{ Main rain season } & Mana-Soda & 0.45 & 0.75 & -0.65 & 0.16 & -0.74 \\
\hline & Surupha & 0.69 & 0.91 & -0.97 & -0.79 & -0.91 \\
\hline & Medhacho & 0.84 & 0.81 & -0.92 & -0.93 & -0.93 \\
\hline & Dambala-Wachu & 0.84 & 0.94 & -0.8 & -0.74 & -0.77 \\
\hline & Did-Tuyura & 0.72 & 0.95 & -0.98 & -0.87 & -0.79 \\
\hline & Bokkulboma & 0.88 & 0.8 & -0.93 & -0.77 & -0.62 \\
\hline & Did-Hara & 0.94 & 0.93 & -0.86 & -0.76 & -0.52 \\
\hline \multirow[t]{7}{*}{ Dry clod season } & Mana-Soda & 0.78 & 0.43 & -0.82 & -0.79 & -0.72 \\
\hline & Dambala-Wachu & 0.83 & 0.81 & -0.95 & -0.94 & -0.92 \\
\hline & Surupha & 0.74 & 0.16 & -0.74 & -0.74 & -0.62 \\
\hline & Medhacho & 0.53 & 0.45 & -0.84 & -0.74 & -0.3 \\
\hline & Bokkulboma & 0.63 & 0.95 & -0.91 & -0.71 & -0.77 \\
\hline & Did-Tuyura & 0.78 & 0.84 & -0.86 & -0.89 & -0.92 \\
\hline & Did- Hara & 0.66 & 0.93 & -0.84 & -0.84 & -0.63 \\
\hline
\end{tabular}




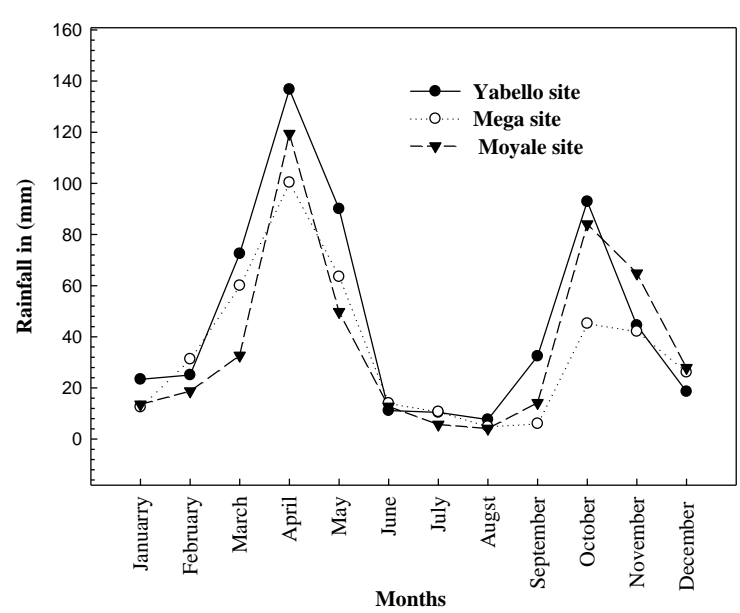

Figure 2. Annual rainfalls for year 2010 in the Borana region, Ethiopia.

rainfall peaks are illustrating the bimodal nature of rainfall in the Borana. The main rain season was between March and May with the peak in April, and the short rain season was between September and November with the peak in October.

Figure 3 presents the seasonal fluctuation of the quality of the common grass species in the study sites. There was a general decline in $\mathrm{CP}$ content from the main rain season towards the cold dry season. Generally, the highest CP content was recorded at Bokkulboma during the main rain season. The structural constituents (i.e., NDF, ADF) of the common grass species showed a slight increase during the cool dry season. The highest value for NDF was recorded during the cold dry season at Did-Tuyura and Mana-Soda. The lowest NDF value was recorded at Bokkulboma in both seasons. The ADF and ash contents of the herbaceous species did not show significant $(p>0.05)$ differences between the two seasons.

\section{Physical and chemical properties of soil}

The textural and chemical properties of soils from the Borana rangelands were significantly different $(p<0.05)$ across the various sites (Table 8). Surupha soils had the highest proportion of sand and the least was recorded at Did-Hara. The silt texture was significantly high $(\mathrm{p}<0.05)$ in soils at Medhacho and least at Surupha. Clay content was highest in soils from Dida-Hara and lowest at Mana-Soda area. The soils in the area were generally alkaline with a mean $\mathrm{pH}$ value of 7. Mana-Soda and Medhacho had the highest $\mathrm{pH}$ values of 8; Surupha and Did-Hara recorded the lowest with values of 5.99 and 6.30, respectively. These $\mathrm{pH}$ values were corroborated by the base metal levels in respective soils. In general, there were variations in the soil chemical constituents across the sites.

The correlation coefficients between the chemical constituents of the herbaceous plants and soil properties are presented in Table 9. The CP content of the common grass species was negatively correlated with the proportion of sandy soils and $\mathrm{Na}^{+}$in the studied soils (Table 9). On the other hand, there was a weak positive correlation between $\mathrm{CP}$, silt, clay and the rest of soil nutrients. A negative correlation between the ash content of herbaceous plants and proportion of sandy soils was also observed. The ADF content of the herbaceous plants was positively correlated with the proportion of clay in the soil. The lignin content of the plants was positively correlated with the $\mathrm{Na}^{+}$content in the study area. Furthermore, the ADL was positively correlated with the proportion of sandy soils, and negatively associated with silt and clay soils. The NDF content was

Table 8. Physical and chemical properties of soil at the different sites

\begin{tabular}{|c|c|c|c|c|c|c|c|c|}
\hline Component & Mana-Soda & Did-Tuyura & Bokkulboma & Surupha & Medhacho & Did-Hara & $\begin{array}{c}\text { Dambala- } \\
\text { Wachu }\end{array}$ & LSD \\
\hline Sand $(\%)$ & $58.73^{\mathrm{b}}$ & $53.33^{\mathrm{bc}}$ & $51.86 \mathrm{c}$ & $71.10^{\mathrm{a}}$ & $57.10^{\mathrm{bc}}$ & $51.30^{c}$ & $58.90^{\mathrm{b}}$ & 10.8 \\
\hline Silt $(\%)$ & $22.10^{\mathrm{ab}}$ & $17.87^{\mathrm{bc}}$ & $22.40^{\mathrm{ab}}$ & $8.40^{\mathrm{d}}$ & $24.70^{\mathrm{a}}$ & $16.70^{\mathrm{c}}$ & $15.90^{c}$ & 7.2 \\
\hline Clay (\%) & $19.20^{\mathrm{d}}$ & $28.80^{\mathrm{ab}}$ & $25.70^{\mathrm{bc}}$ & $20.50^{\mathrm{cd}}$ & $18.30^{\mathrm{d}}$ & $32.00^{\mathrm{a}}$ & $25.20^{\mathrm{bc}}$ & 8.4 \\
\hline pH (meter) & $8.00^{\mathrm{a}}$ & $6.85^{\mathrm{c}}$ & $7.20^{\mathrm{b}}$ & $5.99^{\mathrm{e}}$ & $8.00^{\mathrm{a}}$ & $6.30^{\mathrm{d}}$ & $6.70^{c}$ & 0.2 \\
\hline $\mathrm{Na}^{+}(\mathrm{cmol} / \mathrm{kg})$ & $0.18^{\mathrm{b}}$ & $0.16^{\mathrm{b}}$ & $0.14^{\mathrm{b}}$ & $0.21^{\mathrm{b}}$ & $0.70^{\mathrm{a}}$ & $0.16^{\mathrm{b}}$ & $0.20^{\mathrm{b}}$ & 0.5 \\
\hline $\mathrm{K}^{+}(\mathrm{cmol} / \mathrm{kg})$ & $0.76^{\mathrm{c}}$ & $0.99^{c}$ & $1.35^{\mathrm{b}}$ & $0.28^{\mathrm{d}}$ & $3.10^{\mathrm{a}}$ & $1.39^{\mathrm{b}}$ & $3.10^{\mathrm{a}}$ & 0.4 \\
\hline $\mathrm{Ca}^{+}(\mathrm{cmol} / \mathrm{kg})$ & $27.96^{\mathrm{a}}$ & $13.27^{\mathrm{c}}$ & $14.40^{\mathrm{c}}$ & $4.05^{\mathrm{e}}$ & $25.00^{\mathrm{b}}$ & $9.30^{\mathrm{d}}$ & $6.70^{\mathrm{de}}$ & 4.7 \\
\hline $\mathrm{Mg}^{+}(\mathrm{cmol} / \mathrm{kg})$ & $6.03^{\mathrm{a}}$ & $4.36^{\mathrm{b}}$ & $3.30 b^{c}$ & $0.77^{\mathrm{e}}$ & $6.50^{\mathrm{a}}$ & $2.30^{\mathrm{cd}}$ & $1.70^{\mathrm{de}}$ & 2.2 \\
\hline $\mathrm{CEC}(\mathrm{cmol} / \mathrm{kg})$ & $33.00^{\mathrm{a}}$ & $21.93^{\mathrm{b}}$ & $23.30^{\mathrm{b}}$ & $7.40^{\mathrm{d}}$ & $36.10^{\mathrm{a}}$ & $19.40^{\mathrm{b}}$ & $12.80^{\mathrm{c}}$ & 6.5 \\
\hline $\mathrm{TN}(\%)$ & $0.58^{\mathrm{a}}$ & $0.14^{\mathrm{b}}$ & $0.11^{\mathrm{b}}$ & $0.08^{\mathrm{b}}$ & $0.16^{\mathrm{b}}$ & $0.08^{\mathrm{b}}$ & $0.08^{\mathrm{b}}$ & 0.2 \\
\hline OC (\%) & $1.52^{\mathrm{a}}$ & $1.27^{\mathrm{b}}$ & $1.10^{\mathrm{bc}}$ & $0.89^{\mathrm{c}}$ & $1.40^{\mathrm{a}}$ & $0.80^{\mathrm{a}}$ & $0.80^{\mathrm{c}}$ & 0.5 \\
\hline $\mathrm{OM}(\%)$ & $2.62^{\mathrm{a}}$ & $2.39^{\mathrm{a}}$ & $1.84^{\mathrm{b}}$ & $1.60^{\mathrm{bc}}$ & $2.50^{\mathrm{a}}$ & $1.40^{\mathrm{cd}}$ & $1.10^{\mathrm{d}}$ & 0.6 \\
\hline $\mathrm{C}: \mathrm{N}$ & $9.10^{\mathrm{b}}$ & $9.26^{\mathrm{b}}$ & $10.30^{\mathrm{ab}}$ & $10.70^{\mathrm{a}}$ & $9.10^{\mathrm{b}}$ & $10.90^{\mathrm{a}}$ & $8.90^{\mathrm{b}}$ & 2.2 \\
\hline $\mathrm{P}$ (Av.P/ppm) & $5.81^{\mathrm{c}}$ & $6.17^{\mathrm{c}}$ & $6.20^{\mathrm{bc}}$ & $8.10^{\mathrm{b}}$ & $16.10^{\mathrm{a}}$ & $4.90^{\mathrm{c}}$ & $2.80^{\mathrm{d}}$ & 3.0 \\
\hline
\end{tabular}

Means within a row followed by the same letter were not significantly different ( $\mathrm{p}>0.05)$.

pH: $\mathrm{pH}$ in water; $\mathrm{Na}^{+}$: Sodium; $\mathrm{K}^{+}$: Potassium; $\mathrm{Ca}^{+}$: Calcium; $\mathrm{Mg}^{+}$:Magnesium; CEC: Cation exchange capacity; TN: Total nitrogen; OC: Organic carbon; OM: Organic matter; $\mathrm{C}: \mathrm{N}=$ Carbon to nitrogen ratio; P: Phosphorus, LSD: Least significant difference. 


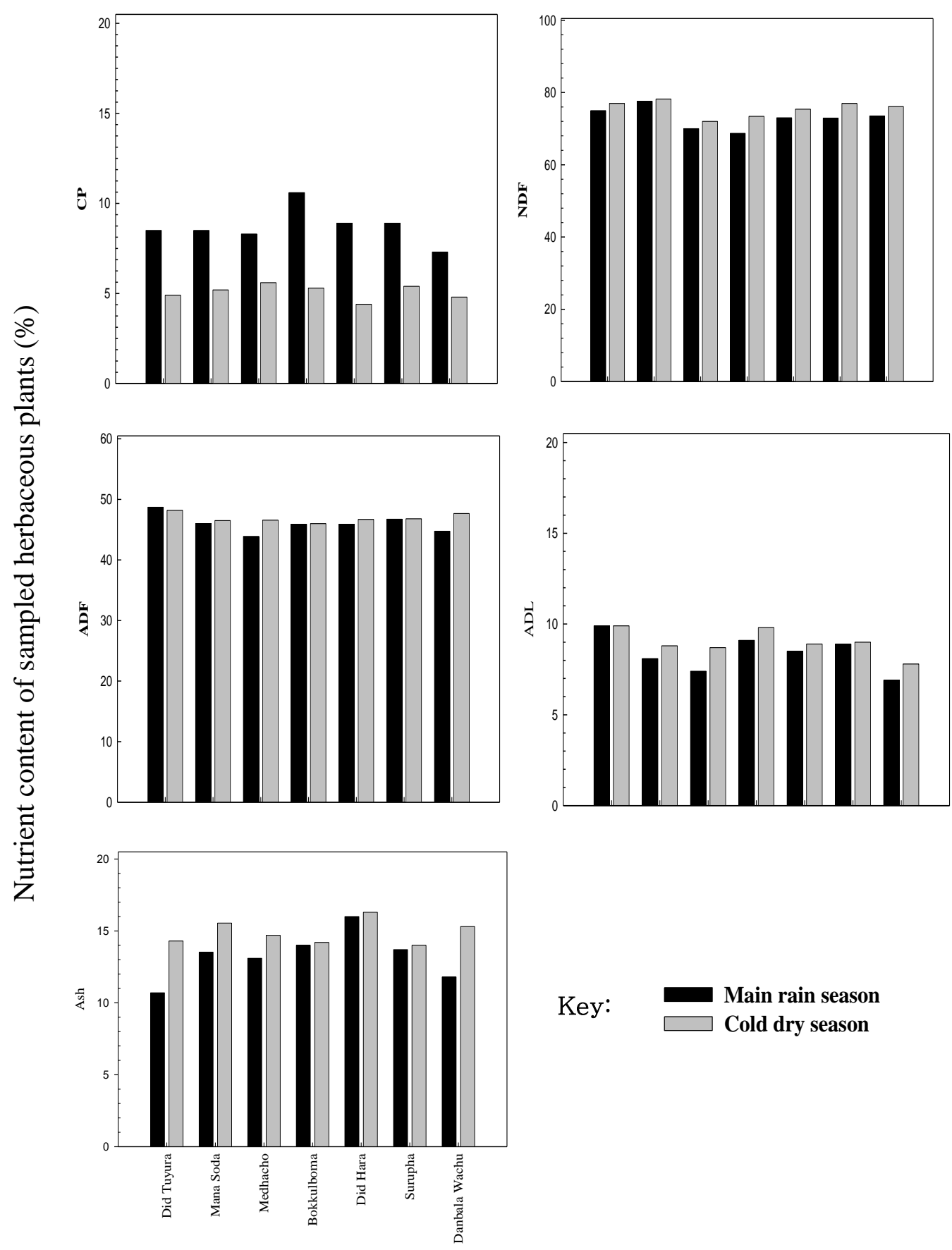

Figure 3. Seasonal and spatial variability of chemical constituents of herbaceous plants in the Borana rangelands.

Table 9. Correlation between chemical constituents of herbaceous plants and soil properties in Borana, southern Ethiopia

\begin{tabular}{lcccccccccccc}
\hline & $\mathrm{pH}$ & Sand & Silt & Clay & $\mathrm{Na}^{+}$ & $\mathrm{K}^{+}$ & $\mathrm{Ca}^{2+}$ & $\mathrm{Mg}^{2+}$ & $\mathrm{CEC}$ & $\mathrm{TN}$ & $\mathrm{OM}$ & $\mathrm{P}$ \\
\hline $\mathrm{CP}$ & 0.293 & $-0.714^{*}$ & 0.429 & 0.333 & $-0.586^{*}$ & 0.333 & 0.429 & 0.238 & 0.333 & 0.206 & 0.143 & -0.143 \\
$\mathrm{NDF}$ & 0.293 & 0.238 & 0.048 & -0.238 & -0.098 & -0.238 & 0.238 & 0.238 & 0.143 & 0.309 & 0.143 & -0.0333 \\
$\mathrm{ADF}$ & -0.195 & -0.333 & 0.048 & $0.524^{*}$ & 0.098 & 0.333 & -0.143 & 0.048 & -0.048 & -0.206 & -0.238 & -0.143 \\
Lignin & 0.05 & 0.195 & -0.098 & -0.293 & $0.550^{*}$ & 0.195 & -0.098 & 0 & 0 & -0.158 & -0.293 & -0.098 \\
Ash & 0.098 & $-0.619^{*}$ & 0.0333 & 0.048 & -0.098 & 0.429 & 0.143 & 0.143 & 0.0238 & -0.103 & -0.143 & -0.048 \\
\hline
\end{tabular}

* Correlation significant $(\mathrm{p} \leq 0.05), \mathrm{CP}=\mathrm{Crude}$ protein, $\mathrm{NDF}=$ neutral detergent fiber, $\mathrm{ADF}=$ acid detergent fiber, $\mathrm{ADL}=$ acid detergent lignin; $\mathrm{pH}: \mathrm{pH}$ in water; $\mathrm{Na}^{+}$: Sodium; $\mathrm{K}^{+}$: Potassium; $\mathrm{Ca}^{+}$: Calcium; $\mathrm{Mg}^{+}$: Magnesium; $\mathrm{CEC}$ : Cation exchange capacity; TN: Total nitrogen; OC: Organic carbon; OM: Organic matter; P: Phosphorus. 
negatively correlated with clay content in the soil but also positively associated with the proportion of sandy and silt soils.

\section{DISCUSSION}

\section{Variation in nutritive value}

Data presented in this study are consistent with the general observations of spatial, seasonal and species variation in terms of the quality of common grass species (Mutanga et al., 2004; Mahala et al., 2009; da Silva, 2011). The CP content of individual grass species was declined as the stage of maturity increases from the main rain season towards the cool dry season. This is largely due to the changes associated with the advancing stage of maturity of individual species (Ammar et al., 1999; Moore and Jung, 2001). Our results are also consistent with published data (e.g. Michiels et al, 2000; Mahala et al., 2009) that suggest that $\mathrm{CP}$ often declines with increasing plant structural constituents (NDF, ADF and lignin) and increasing age of maturity. Ludwig et al. (2004) argued that differences in the quality of forage species are usually attributed to the type of species present, phenology of a species and the aboveground biomass. In the present study, the observed differences in terms of the quality of grass species across sites could probably be explained by the variations in slope, altitude and aspect of the study sites. Similarly, previous studies (Moore and Jung, 2001; Mutanga et al., 2004) have indicated that both the effects of climatic and physical factors are responsible for the variation in the quality of forage species across sites. This is corroborated by documentation of fine-scale redistribution of sediments and nutrients through run-off and run-on under different topographical characteristics (Rietkerk et al., 2002; Ludwig et al., 2005).

Our results indicate that the $\mathrm{CP}$ value for the Borana rangelands is $8.7 \%$ during the main growing season, but reduced to value of $5.1 \%$ in the dry season with an overall average value of $6.9 \%$. This is generally within the recommended range (6 to $8 \%$ ) deemed adequate for maintenance requirements of most wild and domestic herbivores (Ganskop and Bohnert, 2001; Hussain and Durrani, 2009), but lower than the critical limit of $10.6 \%$ proposed by Minson (1990). Using either scale, it is clear that the common grass species could provide inadequate levels of nitrogen for a considerable part of the year.

In addition to soil nutrients, spatial variation is also mediated through variations in soil structure and texture (Mutanga et al., 2004). Although sandy soils are dominant in the Borana region (which is in agreement with reports by Angassa et al., 2012), we recorded a fairly wide range of soil texture across the study sites. This might have the potential of contributing to the variation in plant quality across the study sites. The recorded $\mathrm{pH}$ values in the present study are in agreement with those reported by Angassa et al. (2012). The high content of sandy soils at Surupha might explain the relatively more acidic soils than the other study sites, with significant implications on plant quality. The results of our findings related to the levels of soil exchangeable bases, low $\mathrm{N}$ and organic matter $(\mathrm{OM})$ are in agreement with previous reports (Thompson and Frederick, 1978; Abule et al., 2007). The lowest OM values in our study were recorded at sites grazed heavily on a relative basis. It is likely that the high grazing pressure in the study sites would reduce the available biomass for the process of decomposition in the soil. Angassa et al. (2008) also noted that woody plant encroachment, which is an increasing problem in the study area, leads to low soil OM content. The low $\mathrm{C}$ : $\mathrm{N}$ ratios in the present study are consistent with the limited recyclable plant biomass in arid environments.

The data presented in this study also indicate negative correlations between $\mathrm{CP}$ and proportion of sandy soils and $\mathrm{Na}^{+}$, and a tendency for positive correlation with $\mathrm{K}^{+}, \mathrm{Ca}^{2+}$, $\mathrm{Mg}^{2+}$ and CEC. This and the correlation for ADF, lignin and ash are generally in agreement with the results reported by Gemedo-Dalle (2004). Overall, there is a significant potential for the various biotic and abiotic processes to interact and foster variation in forage quality at both landscape and fine-scale. These interactions are represented by the variations in RFV of a species within and across the study sites.

\section{Grass quality as perceived by pastoralists}

We are providing clear indications that pastoralists are knowledgeable of grass species preferences and palatability by a particular class of livestock. However, there are indications of some disagreements among pastoral communities of different localities. This inconsistency of LEBHP perception was also noted in other studies elsewhere (Knapp and Fernendez-Gimenez, 2008; Kassam, 2009). Given that the study sites had spatial variation in topography, land-use patterns and soil characteristics, there was bound to be heterogeneity in species composition and their relative abundances. Different communities would therefore develop LEBHP perceptions on species prevalent in their particular area.

In general, the ranking values of common grass species by our respondents was strongly correlated with chemical composition values obtained from the laboratory analysis. The correlations were positive for $\mathrm{CP}$ and ash, but negative for $\mathrm{NDF}, \mathrm{ADF}$ and $\mathrm{ADL}$. The variations in correlation coefficients between species' ranking values and their chemical composition again indicate inconsistency of the LEBHP perceptions among the different communities groups across the study sites and spatial variation in plant constituents. Overall, our data demonstrate the ranking value of LEBHP perception in the assessments of the quality of common grass species in Borana. In the past, such knowledge has largely been overlooked by researchers (Pierotti and Wildcat, 2000). Thus, there is a potential for 
the integration of LEBHP evaluation and laboratory analysis to complement each other in rangeland assessments in future development endeavors.

\section{CONCLUSIONS}

The chemical constituents of herbaceous plants in the Borana rangelands showed significant site, season and species variation. Although soil texture and chemical properties influenced the quality of the grass species, advances in the stage of plant maturity and climate variability in general were critical in determining the quality of grass species in Borana. The CP content was greatly declined with advances in plant maturity and associated climatic condition. Crude protein was also negatively correlated with soil $\mathrm{Na}^{+}$content. The fiber constituents were increased markedly as the stage of plant maturity increased towards the dry season and showed a positive correlation with $\mathrm{Na}^{+}$. There were a positive and strong correlation between the LEBHP perception and laboratory based constituents of a particular species. Our results from laboratory analysis of species confirmed that grasses ranked as superior by the pastoralists also had higher CP than those species perceived as inferior. Generally, grass species categorized as low palatability had high structural fiber levels as confirmed by the laboratory analysis. Such correlations indicate the potential of these assessment methods to complement each other in future research strategies.

\section{ACKNOWLEDGEMENTS}

The authors acknowledge the Federal Democratic Republic of Ethiopia, Ministry of Agriculture for the financial support through Rural Capacity Building Project (RCBP); Debrezeit Agriculture Research Center for laboratory analysis of the plant samples; and, Oromia Water Works Design and Supervision Enterprise for handling soil chemical analysis. We also thank the two anonymous referees for their helpful comments to improve the quality of this manuscript.

\section{REFERENCES}

Abule, E., H. A. Snyman and G. N. Smit. 2007. Rangeland evaluation in the middle Awash valley of Ethiopia: III. Relationships among soil and vegetation variables. J. Arid Environ. 70: 293-303.

Alemayehu, M. 2006. Range Management for Eastern Africa: Concepts and Practices. Addis Ababa University Printing Press. Addis Ababa.

Ammar, H., S. Lopez, O. Bochi-Brum, R. Garcua and M. J. Ranilla. 1999. Composition and in vitro digestibility of leaves and stems of grasses and legumes harvested from permanent mountain meadows at different stages of maturity. J. Anim. Feed Sci. 8: 599-610.

Angassa, A. and G. Oba. 2007. Relating long term rainfall variability to cattle population dynamics in commonual rangelands and government ranch in southern Ethiopia. Agr. Syst. 94: 715-725

Angassa, A. and G. Oba. 2008. Effects of management and time on mechanisms of bush encroachment in southern Ethiopia. Afr. J. Ecol. 46: 186-196.

Angassa, A.and G. Oba. 2010. Effect of grazing pressure, age of enclosures and seasonality on bush cover dynamics and vegetation composition in Southern Ethiopia. J. Arid Environ. 74: 111-120.

Angassa, A. and B. Sheleme, G. Oba, A. C. Trendte, A. Linstadter and J. Sauerborn. 2012. Savanna land use and its effect on soil characteristics in Southern Ethiopia. J. Arid Environ. 81: 67-76..

AOAC. 1990. Association Official Analytical Chemists $13^{\text {th }}$ ed. Official methods of analysis, Washington DC, USA.

Arzani, H., M. Basiri, F. Khatibi and G. Ghorbani. 2006. Nutritive value of some Zagros Mountain rangeland species. Small Rumin. Res. 65:128-135.

Arzani, H., M. R. Sadeghimanesh, H. Azarnivad, G. H. Asadian and E. Shahriyari. 2008. Study of phonological stages effect values of twelve species in Hamadan rangelands. Iran J. Range Desert Res. 16: 86-95.

Beever, D. E., N. Offer and E. M. Gill. 2000. The feeding value of grass and grass products. In: Grass (Ed. A. Hopkins). Its production and utilization. Third edition. Blackwell Science Ltd. UK. pp. 140-195.

Coppock, D. L. 1994. The Borana Plateau of southern Ethiopia. Synthesis of pastoral Research, development and change, 1980-91. International livestock center for Africa (ILCA) ,Addis Ababa, Ethiopia, p. 299.

Da Silva, D. S., M. V. M. de Andrade, A. P. de Andrade, M. S. de Souza Carneiro and J. S. de Oliveira. 2011. Bromatologic composition of the herbaceous species of the North eastern Brazil Caatinga. Rev. Bras. Zootech. 40:756-764.

Fowler, J. and L. Cohen. 1996. Practical Statistics for Field Biology. John Wiley and Sons, Chichester, UK.

Ganskopp, D. and D. Bohnert. 2001. Nutritional dynamics of seven northern Great basin grasses. J. Range. Manage. 54: 640-647.

Gee, G. W. and J. W. Bauder. 1986. Particle size analysis. In: methods of soil analysis, part 1. Physical and mineralogical methods (Ed. A. Klute). Agronomy Monograph (2ed) Am. Soc. Agron. 9:383-411.

Gemedo, D. 2004. Vegetation ecology, rangeland condition and forage resources evaluation in the Borana Lowlands, Southern Oromia, Ethiopia. A PhD Thesis, University of Gotingen, Germany.

Gemedo, D., B. L. Maass and J. Isselstein. 2006. Encroachment of woody plants and its impact on pastoral livestock production in the Borana lowlands, southern Oromia, Ethiopia. Afr. J. Ecol. 44:237-246.

Henkin, N., E. D. Ungar, L. Dvash, A. Perevolotsky, Y. Yehuda, M. Sternbergs, H. Voet and S. Y. Landau. 2011. Effects of cattle grazing on herbage quality in a herbaceous Mediterranean rangeland. Grass Forage Sci. 66:516-525. 
Hussain, F. and J. D. Mufakhirah. 2009 Nutritional evaluation of some forage plants from Harboi rangeland, Kalat, pakstan. Pak. J. Bot. 41:1137-1154.

Kassam, K. 2009. Viewing change through the prism of indigenous human ecology: Findings from the Afghan and Tajik Paamirs. Human Ecol. 37:677-690.

Knapp, C. N. and M. Fernandez-Gimenez. 2008. Knowing the land: A review of local knoweladge revealed in the ranch memoirs. Rangeland Ecol. Manage. 61:148-155.

Ludwing, F., H. de Kroon, F. Berendse and H. H. T. Prins. 2004. The influence of savanna trees on nutrient, water and light availability and the under storey vegetation. Plant Ecol. 170: 93-105.

Ludwing, J. A., B. P. Wilcox, D. D. Breshears, D. J. Tongway and A. C. Imeson. 2005. Vegetation patches and run-off-erosion as interacting ecohydrological processes in semi-arid landscapes. Ecol. 86:288-297.

Mahala, A. G., I. V. Nsahlai, N. A. D. Basha and L. A. Mohammed. 2009. Nutritive evaluation of natural pasture at early and late rainfall season in Kordofan and Butana, Sudan, Aust. J. Basic Appl. Sci. 3:4327-4332.

McLean, E. O. 1982. Soil pH and lime requirement. In: Methods of soil analysis (Ed. A. L. R. Page, R. H. Miller and D. R. Keeney). part 2. Chemical and microbiological properties $\left(2^{\text {nd }}\right.$ ed.) Agronomy 9:199-223.

Michiels, B., S. Babatounde, M. Dahouda, S. L. W. Chabi and A. Buldgen. 2000. Botanical composition and nutritive value of forage consumed by sheep during the rain season in a Sudan Guinean savanna (central Benin). Trop. Grasslands 34:43-47.

Minson, D. J. 1989. The chemical composition and nutritive value of tropical grasses. In: XTropical grasses. FAO plant and production and protection series No. 23 (Ed. P. J. Skerman and F. Riveros). Food and Agriculture Organization of the United Nations. Rome, Italy, pp. 133-162.

Minson, D. J. 1990. Forage in ruminant nutrition. (Ed. T. J. Conha). pp. 60-263. Academic Press, Inc., Javanorich Publishers, USA.

Moore, K. J. and H. J. Jung. 2001. Lignin and fiber digestion. J. Range. Manage. 54:420-430.

Mutanga, O., H. H. T. Prins, A. K. Skidmore, S. van Wieren, H. Huizing, R. Grant, M. Peel and H. Biggs. 2004. Explaing grass nutrient patterns in savannah rangeland of southern Africa. J. Biogeogr. 31:819-829.

Oba, G. 1998. Assessments of indigenous range management knowledge of the Borana pastoralists in southern Ethiopia. Borana Lowland Pastoral Development Program/GTZ, Consultancy paper, Negelle /Borana May 1998.

Oba, G. and D. G. Kotile. 2001. Assessments of landscape level degradation in southern Ethiopia: Pastoralists versus ecologists. Land Degrad. Dev. 12:461-475.
Oba, G., R. B. Weladji, D. J. Msangameno, L. M. Kaitira and J. Stave. 2008. Scaling effects of proximate desertification drivers on soil nutrients in northeastern Tanzania. J. Arid Environ. 72:1820-1829.

Oddy, V. U., G. E. Roberts and S. G. Low. 1983. Prediction of in-vivo dry matter digestibility from fiber and nitrogen content of feed. Common Wealth Agriculture Bureax, Australia. pp. 295-298.

Olsen, S. R. and L. E. Sommers. 1982. Phosphorus. In: Methods of Soil Analysis (Ed. A. L. Page, R. H. Miller, D. R. Keeney). Part 2: Chemical and Microbiological Properties. J. Am. Soc. Agron. 403-427.

Pierotti, R. and D. Wildcat. 2000. Traditional ecological knoweladge: The third alternative (Commentary). Ecol. Appl. 10:1333-1340.

Rietkerk, M., T. Ouedraogo, L. Kumar, S. Sanou, J. van Andel, J. Hearne, A. K. Skidmore, N. de Ridder, L. Stroosnijder and H. H. T. Prins. 2002. Fine-Scale spatial distribution of plants and resources on sandy soil in Sahel. Plant and Soil 239:69-77.

SAS Institute. 2001. Statistical Analysis Software. SAS Institute Inc., Cary, NC, USA.

SPSS, 2003. Statistical procedure for social science version 17. Statistical procedures for social sciences (SPSS) INC, Chicago, USA.

Snyman, H. A. 1998. Dynamics and sustainable utilization of the rangeland ecosystem in arid and semi-arid climates of southern Africa. J. Arid Environ. 39:645-666.

Stalling, C. C. 2005. Test available for measuring forage quality. Virginia Cooperative Extension Dairy Guideline 404-124. http://pubs.ext.vt.edu./404/404-124/404-124html.

Stoddart, L. A., A. D. Smith and T. W. Box. 1975. Range Management. New York, Mc Grew-Hill. p. 532.

Tessema, Z. K., W. F. de Boer, R. M. T. Baars and H. H. T. Prins. 2011. Changes in soil nutrients, vegetation structure and herbaceous biomass in response to grazing in a semi-arid savanna of Ethiopia. J. Arid Environ. 75:662-670.

Thompson, L. M. and R. T. Frederick. 1978. Soil and Soil Fertility. McGraw-Hill Publishing Company, New York, USA.

Van der Westhuizen, H. C., H. A. Snyman and H. J. Fouchè. 2005. A degradation gradient for the assessment of rangeland condition of a semi-arid sour veld in southern Africa. Afr. J. Range Forage Sci. 22:47-58.

Van Reeuwijk, L. P. 1995. Procedures for Soil Analysis. Technical paper 9, fifth ed. ISRIC, Wageningen.

Van Soest, P. J., J. B. Robertson and B. A. Lewis. 1991. Methods for dietary fiber, neutral detergent fiber and non - starch polysaccharides in relation to animal nutrition. J. Dairy Sci. 74:3583-3597. 